\title{
MAPS AND DIAGRAMS
}

Europe $c .155^{8} \quad$ xii

The Population of Europe $c$. 1600

Economic Features of Europe c. $155^{8}$

Spanish Imports of Treasure $\quad{ }_{136}$

The Dutch Revolt $\quad{ }_{166}$

The Distribution of Huguenot Strength and the Growth of the Places de Sûreté c. I6oo 180

England and the Civil War 216

The Thirty Years' War, $1618-1648 \quad 256$ 
